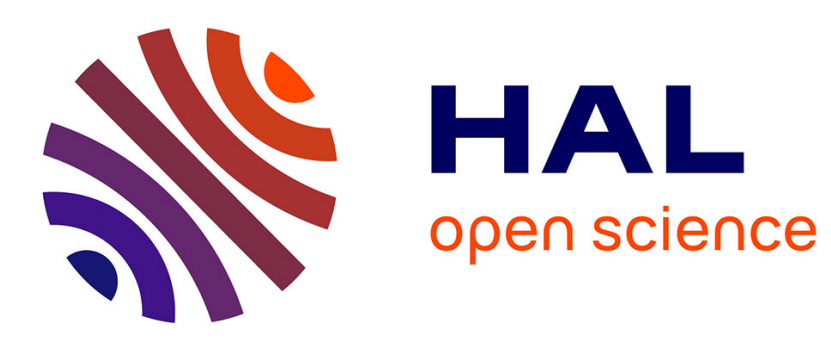

\title{
Experimental Evaluation of a Software Defined Visible Light Communication System
}

\author{
Bassam Aly, Mohammed Elamassie, Burak Kebapci, Murat Uysal
}

\section{To cite this version:}

Bassam Aly, Mohammed Elamassie, Burak Kebapci, Murat Uysal. Experimental Evaluation of a Software Defined Visible Light Communication System. 2020 IEEE International Conference on Communications Workshops (ICC Workshops), Jun 2020, Dublin, Ireland. pp.1-6, 10.1109/ICCWorkshops49005.2020.9145145 . hal-03342320

\section{HAL Id: hal-03342320 https://hal-amu.archives-ouvertes.fr/hal-03342320}

Submitted on 13 Sep 2021

HAL is a multi-disciplinary open access archive for the deposit and dissemination of scientific research documents, whether they are published or not. The documents may come from teaching and research institutions in France or abroad, or from public or private research centers.
L'archive ouverte pluridisciplinaire $\mathbf{H A L}$, est destinée au dépôt et à la diffusion de documents scientifiques de niveau recherche, publiés ou non, émanant des établissements d'enseignement et de recherche français ou étrangers, des laboratoires publics ou privés. 


\title{
Experimental Evaluation of a Software Defined Visible Light Communication System
}

\author{
Bassam Aly ${ }^{*, \dagger}$, Mohammed Elamassie ${ }^{\dagger}$, Burak Kebapci ${ }^{\dagger, \ddagger}$, and Murat Uysal ${ }^{\dagger}$ \\ ${ }^{*}$ R\&D Department, Ford Otosan, Istanbul, Turkey, 34885. \\ ${ }^{\dagger}$ Department of Electrical and Electronics Engineering, Özyeğin University, Istanbul, Turkey, 34794. \\ ${ }^{\ddagger}$ Hyperion Technologies, Istanbul, Turkey, 34794. \\ E-mails: bmohamed@ford.com.tr, mohammed.elamassie@ozu.edu.tr, \\ burak.kebapci@hyperiontechs.com, murat.uysal@ozyegin.edu.tr.
}

\begin{abstract}
Visible light communication (VLC) allows the dual use of light-emitting diodes (LEDs) for illumination and communication purposes. With its large bandwidth and immunity to electromagnetic interference, VLC can be used as complementary and/or alternative to radio communications. In this paper, we present a comprehensive experimental evaluation of a softwaredefined VLC system with on-off keying for both line-of-sight (LOS) and non-line-of-sight (NLOS) scenarios. The experimental set-up is based on a pair of modified National Instruments USRPs coupled with a custom design front-end. In the measurement campaign, we vary the distance between 1 to 3 meters with a step size of $0.5 \mathrm{~m}$. For each point in this LOS scenario, we measure signal-to-noise ratio (SNR) and bit error rate (BER) with and without a lens. We also evaluate the effect of the reception angle (i.e, no strict alignment) on system performance. We further evaluate the SNR performance for NLOS scenarios and demonstrate a robust performance due to the wide field-ofview of the front-end.
\end{abstract}

Index Terms-Visible light communication, software-defined, USRP, on-off keying, estimated SNR, LOS, NLOS, indoor measurements.

\section{INTRODUCTION}

Visible light communication (VLC) allows the dual use of light-emitting diodes (LEDs) for illumination and communication purposes [1]. With its large bandwidth and immunity to electromagnetic interference, VLC has emerged as powerful wireless access technology. Particularly, VLC has a robust performance in user-dense environments and is considered as a complementary technology to WiFi via data offloading. Furthermore, VLC can be used as a safe alternative in radiofrequency (RF) restricted areas such as hospital intensive care units, nuclear plants etc. [2].

VLC is based on modulating the light intensity of LEDs. In a VLC system, the information is superimposed upon the direct current (DC) driving the LED [2]. The DC value is selected according to the desired operating point taking into account the amplitude constraints. Since the frequency of the

The work of Bassam Aly was supported by the European Horizon 2020 MSC ITN (VISION) under Grant 764461. The work of M. Uysal was supported by the Turkish Scientific and Research Council (TUBITAK) under Grant 215E311. modulating signal is very high, the flickering of the light intensity cannot be observed by the human eye. Typical modulation choice for VLC systems is on-off keying (OOK) and pulse modulation techniques while other modulation techniques such as orthogonal frequency division multiplexing (OFDM) have been further proposed to support ultra-high-speeds on the order of Gbps.

There is already a rich literature on theoretical aspects of VLC, see e.g., a recent survey [3] and references therein, covering various aspects of channel modelling, physical layer design, and networking. The performance of VLC systems was also evaluated by experimental means. For example, in [4], a VLC system with OOK non-return zero (OOK-NRZ) modulation was considered and its bit error rate (BER) was measured at $2 \mathrm{~m}$ distance. In [5], the performance of an OFDM-based VLC system was experimentally evaluated for different modulation orders. An arbitrary waveform generator was used to generate OFDM signals while the signal decoding was performed offline with MATLAB. In [6], OFDM-based VLC transmitter and receiver were implemented on FPGA and its performance was evaluated at a distance of $2 \mathrm{~m}$. While most of these studies assumed line-of-sight (LOS) scenarios, the performance of VLC systems was further demonstrated in non-line-of-sight (NLOS) conditions [7]-[9]. For example, the channel impulse response of VLC channel was characterized in [7] using a vector network analyzer and it was reported that the received power associated with NLOS components were 25-37 dB less than that of LOS at the same distance [7]. In [8], path loss was measured for a NLOS scenario using a blue LED. The transmitter and receiver were fixed at $1.815 \mathrm{~m}$ and $1.716 \mathrm{~m}$, respectively, below the ceiling. The received optical power at different horizontal separations was measured while orienting the receiver to the maximum received value. The path loss was $41 \mathrm{~dB}$ at 1 meter and increased to $53 \mathrm{~dB}$ at $6 \mathrm{~m}$. In [9], a vector signal analyzer was used to generate 16 QAM and the impact of receiver angles on the VLC system performance was investigated. For $40 \mathrm{~cm}$ distance between transmitter and reflector (print paper as a reflector), the received power was measured as $-15.2 \mathrm{dBm}$ for a reflecting angle of $10^{\circ}$.

In addition to the above experiments conducted mainly with laboratory equipment or dedicated hardware designs on FPGA or microcontrollers, software-defined platforms, as a 


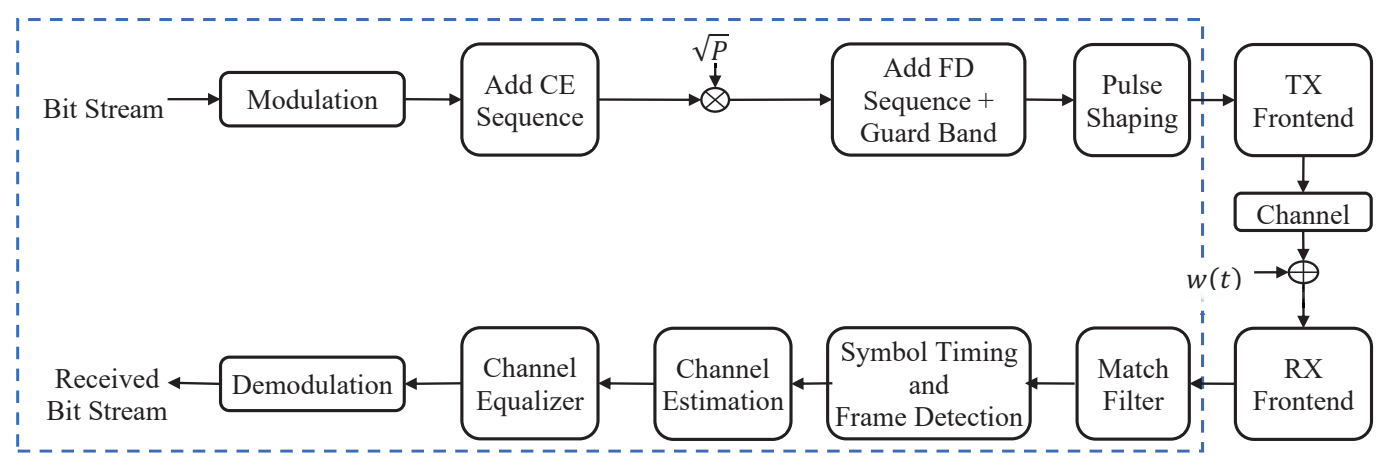

Fig. 1. Block diagram of VLC system under consideration.

more flexible prototyping tool, were used in VLC experiments. For instance, in [10], a modified USRP (Universal Software Radio Peripheral) from National Instruments (NI) was integrated with an electro-optical front-end built with offthe-shelf components. This set-up was used to experimentally evaluate different modulations of IEEE 802.15.7 standard (i.e., Manchester encoded OOK and 4B6B encoded PPM). In [11], a similar experiment with USRPs was conducted using properly DC-biased binary phase-shift keying and a data rate of 2.5 Mbps was obtained at a distance of $160 \mathrm{~cm}$.

In this paper, we present a comprehensive experimental evaluation of a software-defined VLC system with OOK considering both LOS and NLOS scenarios. The experimental set-up is based on a pair of modified NI USRPs where RF daughterboards are replaced by baseband LFTX and LFTX cards [12]. These are coupled with a custom design frontend recently made available by Hyperion Technologies [13]. This front-end has an electrical bandwidth of $20 \mathrm{MHz}$ and includes integrated pre/post-equalizers. The receiver has a wide field-of-view of 170 degrees and supports both LOS and NLOS connectivity. In the measurement campaign, we make measurements for SNR and BER. We further investigate the effect of using lens and receiver/transmitter orientation on system performance.

The rest of this paper is organized as follows. In Section II, we describe the system model. In Section III, we discuss frame detection, symbol timing, DC bias estimation and channel estimation which are essential for practical implementation. In Section IV, we present the experimental setup and measurement results. Finally, the paper is concluded in Section V.

\section{SYSTEM MODEL}

As illustrated in Fig. 1, we consider a VLC system with OOK. Let $S(i), i=1, \cdots, L_{\mathrm{D}}$ denote the $i^{\text {th }}$ transmitted OOK symbol. The continuous-time waveform can be expressed as

$$
x(t)=\left(\sqrt{P_{\mathrm{t}}} \sum_{i=1}^{L_{D}} S(i) \delta\left(t-i T_{s}\right)\right) \otimes p_{T}(t),
$$

where $p_{T}(t), T_{s}$ and $\delta(\cdot)$ denote, respectively, transmit pulse shaping filter, pulse duration and Dirac delta function. Here, $P_{\mathrm{t}}$ denotes the transmit power. It is set as $P_{\mathrm{t}}=0$ if bit ' 0 ' is transmitted, otherwise set as $P_{\mathrm{t}}=2 P$. Therefore, the average electrical transmit power becomes $P$. After adding a DC bias $\left(V_{D C}\right)$, the waveform that drives the LED can be written as

$$
x_{\mathrm{LED}}(t)=A\left(\sqrt{P_{\mathrm{t}}} \sum_{i=1}^{L_{D}} S(i) \delta\left(t-i T_{s}\right)\right) \otimes p_{T}(t)+V_{D C},
$$

where $A$ is an amplification factor. The signal goes through the optical channel. At the receiver side, a photodetector captures the light and converts into an electric current. The received electrical signal at the destination can be written as

$$
x_{\mathrm{PD}}(t)=\left[R x_{\mathrm{LED}}(t) \otimes l(t) \otimes h(t)+w(t)\right] \otimes p_{\mathrm{R}}(t),
$$

where $R$ is the photodetector responsivity, $l(t)$ is the LED impulse response, $h(t)$ is the optical channel impulse response, $p_{R}(t)$ is the receive pulse-shaping filter, and $w(t)$ is the additive white Gaussian noise term with mean $\mu_{n}$ and noise variance of $\sigma_{n}^{2}$. Replacing (2) in (3), we have

$$
\begin{aligned}
x_{\mathrm{PD}}(t)= & \left(\sqrt{P_{\mathrm{t}}} \sum_{i=1}^{L_{D}} S(i) \delta\left(t-i T_{s}\right)\right) \otimes h_{e f f}(t) \\
& +V_{R_{D C}}+w_{g}(t),
\end{aligned}
$$

where $w_{g}(t)=w(t) \otimes p_{R}(t)$ and $V_{R_{D C}}=R V_{D C} \otimes l(t) \otimes$ $h(t) \otimes p_{R}(t)$. It can be readily checked that the statistics of $w_{g}(t)$ remain the same as those of $w(t)$. In (4), we define $h_{\text {eff }}(t)=A R p_{T}(t) \otimes l(t) \otimes h(t) \otimes p_{R}(t)$ as the "effective channel impulse response" incorporating the effects of propagation channel, front-ends, and pulse shaping. After sampling the received signal, the $i^{\text {th }}$ sample can be expressed as

$$
\begin{aligned}
x_{\mathrm{PD}}(i)= & \sqrt{P_{\mathrm{t}}} S(i) h_{e f f}(i)+V_{R_{D C}} \\
& +w_{g}(i), \quad i=1, \cdots, L_{\mathrm{D}}
\end{aligned}
$$

where $w_{g}(i), x_{\mathrm{PD}}(i)$ and $h_{e f f}(i)$ denote, respectively, the $i^{\text {th }}$ sample of $w_{g}(t), x_{\mathrm{PD}}(t)$ and $h_{e f f}(t)$. For detection, we first remove the effect of DC bias. Mathematically speaking, we have

$$
\widehat{x_{P D}}(i) \approx \sqrt{P_{\mathrm{t}}} h_{e f f} S(i)+\left(V_{R_{D C}}-\widehat{V_{R_{D C}}}\right)+w_{g}(i) .
$$




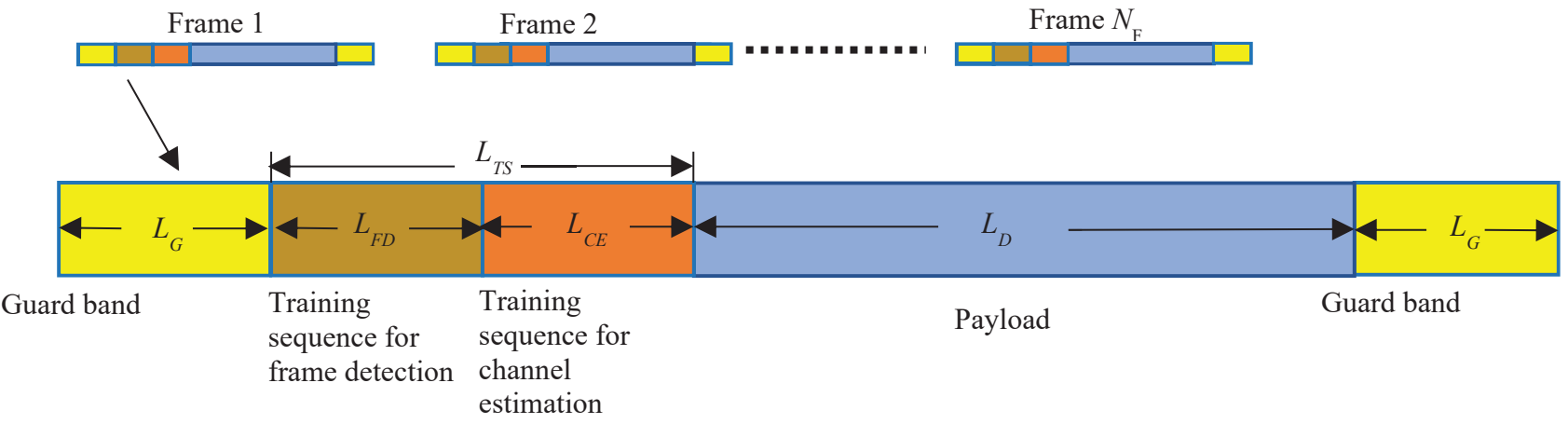

Fig. 2. Frame structure.

where $\widehat{V_{R_{D C}}}$ is the estimated value of DC-bias (see Section III). Using a single-tap equalizer, we can retrieve the message signal as

$$
\begin{aligned}
\widehat{S}(i) & \approx\left(\sqrt{P_{\mathrm{t}}} \widehat{h_{e f f}}\right)^{-1} \widehat{x_{P D}}(i) \\
& =\frac{h_{e f f}}{\widehat{h_{e f f}}} S(i)+\widehat{w}(i),
\end{aligned}
$$

where $\widehat{w}(i)=\left(\left(V_{R_{D C}}-\widehat{V_{R_{D C}}}\right)+w(i)\right) / \sqrt{P_{\mathrm{t}}} \widehat{h_{e f f}}$ and $\widehat{h_{e f f}}$ is the estimate of the channel coefficient (see Section III).

\section{PRACTICAL IMPLEMENTATION ASPECTS}

In this section, we discuss frame detection, symbol timing, DC bias estimation and channel estimation which are essential for practical implementation. The frame structure is illustrated in Fig. 2. Each frame includes a guard band of length $L_{G}$ followed by a training sequence with a length of $L_{\mathrm{TS}}=L_{F D}+L_{C E}$. The training sequence is used for three purposes: i) Frame detection, ii) DC bias estimation and iii) channel estimation. This is then followed by the transmission of $L_{D}$ data symbols. Finally, another guard band with a length of $L_{G}$ is inserted before the transmission of the next frame. Therefore, a frame has a total length of $L_{\text {down }}=2 L_{\mathrm{G}}+L_{\mathrm{TS}}+L_{\mathrm{D}}$. Root-raised-cosine (RRC) with a roll-off factor of $\beta$ is used as a transmitter pulse shaping filter. After pulse shaping, the total number of samples in a transmitted frame is given by $L_{\text {up }}=L_{\text {down }} U$ where $U$ denotes the up-sampling factor.

First, we perform symbol timing to estimate the sampling point of the symbol. For this purpose, we use the maximum output energy method [14]. This finds the sample point where the average received energy is maximized [14, Chapter 5]. Mathematically speaking, this can be obtained as

$$
\hat{\tau}=\max _{\tau}\left|E\left[\left(Y_{u p}(i U+\tau)\right)^{2}\right]\right|, i=1,2, \cdots, L_{\text {down }}
$$

where $Y_{\text {up }}(i), i=1,2, \cdots, L_{\text {up }}$ denote the received samples and $\tau=1,2, \cdots, U$ denote the possible delay values.
After symbol timing, frame detection is performed in order to resolve multiple symbol period delays [15]. The training sequence for frame detection consists of ones and zeros. Based on (6), the corresponding received samples can be expressed as

$$
\begin{gathered}
x_{\text {zeros }}(i)=V_{R_{D C}}+w_{g}(i), \quad i=1, \cdots, L_{\text {zeros }} \\
x_{\text {ones }}(i)=\sqrt{P_{\mathrm{t}}} h_{e f f}+V_{R_{D C}} \\
+w_{g}(i), \quad i=1, \cdots, L_{\text {ones }}
\end{gathered}
$$

where $L_{\text {zeros }}$ and $L_{\text {ones }}=L_{F D}-L_{\text {zeros }}$, respectively, denote the numbers of zeros and ones used for training. For DCbias estimation, we calculate the average of (9) over available samples which yields

$$
\widehat{V_{R_{D C}}}=\frac{1}{L_{\text {zeros }}} \sum_{i=1}^{L_{\text {zeros }}} x_{\text {zeros }}(i) .
$$

After removing the estimated DC-bias, we can then express (9) and (10) as

$$
\begin{aligned}
x_{\text {zeros-DC }}(i) & =w_{g-\mathrm{DC}}(i), \quad i=1, \cdots, L_{\text {zeros }} \\
x_{\text {ones-DC }}(i) & =\sqrt{P_{\mathrm{t}}} h_{e f f} \\
& +w_{g-\mathrm{DC}}(i), \quad i=1, \cdots, L_{\text {ones }}
\end{aligned}
$$

where $w_{g \text {-DC }}(i)=\left(V_{R_{D C}}-\widehat{V_{R_{D C}}}\right)+w_{g}(i)$ denotes the effective noise. It can be readily verified that, theoretically, the effective noise is still zero-mean Gaussian with a variance of $\sigma_{n}^{2}$. Utilizing (12), we can estimate the noise power of the effective noise as

$$
\widehat{\sigma_{n}^{2}}=\frac{1}{L_{\text {zeros }}} \sum_{i=1}^{L_{\text {zeros }}}\left|x_{\text {zeros-DC }}(i)\right|^{2} .
$$

On the other hand, using (13), we can estimate the effective channel coefficient as

$$
\widehat{h_{e f f}}=\frac{1}{\sqrt{P_{\mathrm{t}}} L_{\mathrm{ones}}} \sum_{l=1}^{L_{\mathrm{ones}}} x_{\mathrm{ones}-\mathrm{DC}}(i) .
$$


TABLE I

EXPERIMENTAL SET-UP PARAMETERS

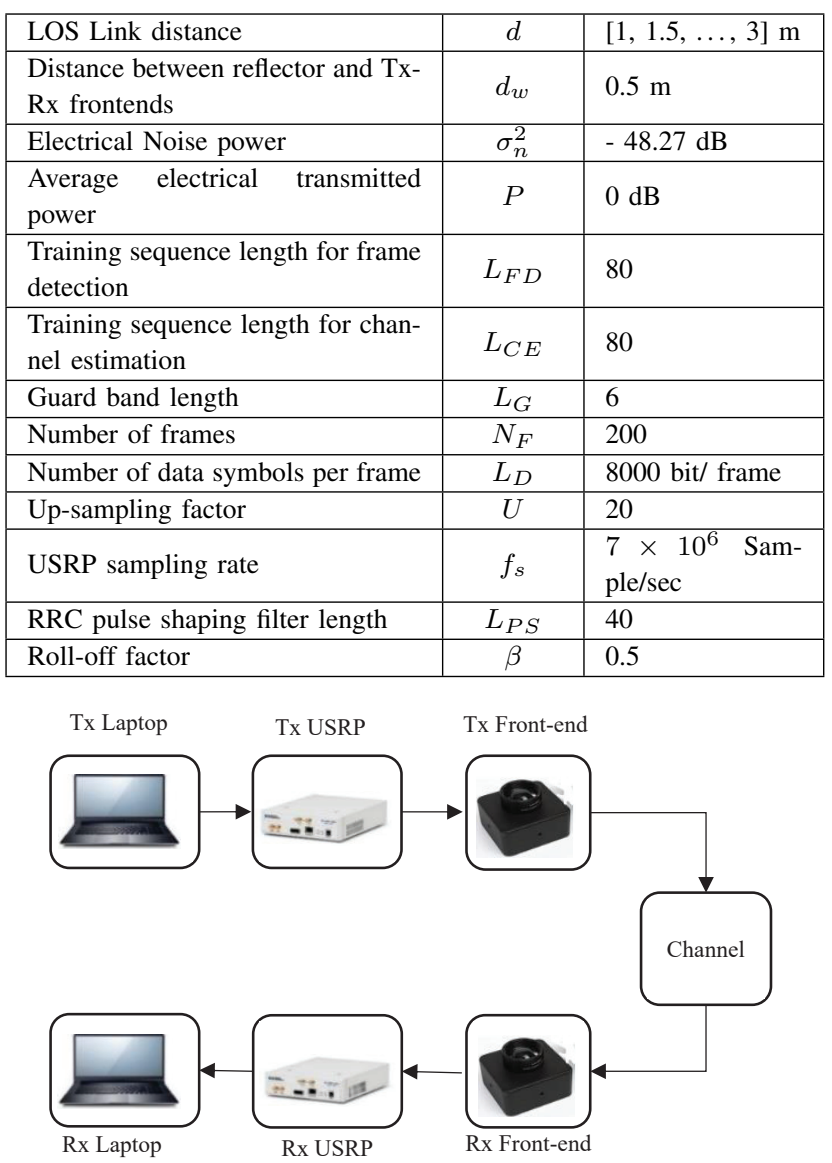

Fig. 3. Block diagram of the experimental set-up.

Based on (14) and (15), estimated SNR can be written as

$$
S \widehat{N R}[k]=\frac{P_{\mathrm{t}}{\widehat{h_{e f f}}}^{2}}{\widehat{\sigma_{n}^{2}}} \text {. }
$$

\section{EXPERIMENTAL SETUP AND RESULTS}

Fig. 3 illustrates the block diagram of our experimental setup. At the transmitter side, the laptop with LabVIEW software generates the digital signal which is transferred to the modified NI 2920 USRP with LFTX daughterboard through an ethernet cable [11]. After digital-to-analogue conversion (DAC), the signal is delivered to custom-design VLC frontend [12]. Tx front-end is equipped with a Lambertian pattern LED with 60 degrees half angle. Tx front-end has $20 \mathrm{MHz}$ and interchangeable lens mechanism. The optical signal propagates through the channel and captured by the receiver front-end. The Rx front-end has a wide FOV of 170 degree which allows NLOS operation [12]. Both front-ends are compatible with USRPs and PXIs. This is followed by another modified NI 2920 USRP with LFRX daughterboard. The output of USRP is a digital signal which is fed to the receiver laptop for demodulation.

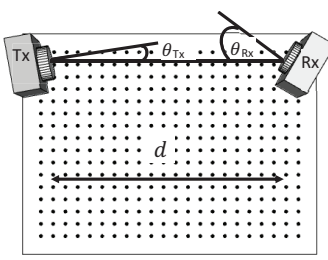

(a)

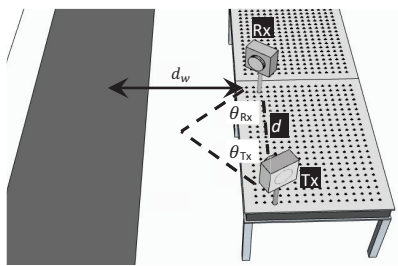

(b)
Fig. 4. Experimental scenarios: (a) LOS (b) NLOS.
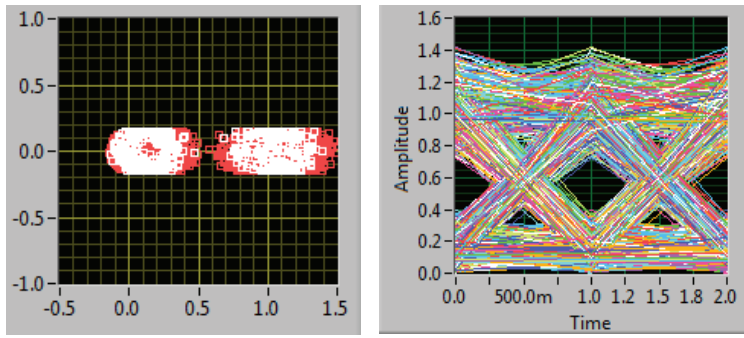

(a)
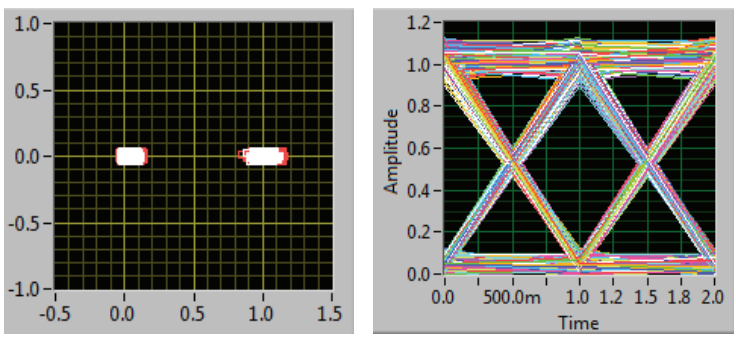

(b)

Fig. 5. Constellation and eye diagram of received signal at 1 meter in LOS scenario with strict alignment (a) without lens (b) with lens.

In our measurement campaign, we consider both LOS and NLOS scenarios as illustrated in Fig. 4. The length of the straight line between transmitter and receiver is denoted by $d$. $\theta_{\mathrm{TX}}$ and $\theta_{\mathrm{RX}}$ respectively define the orientation of transmitter and receiver. In the LOS scenario (see Fig. 4.a), we consider two cases: a) strict alignment between $\mathrm{Tx}$ and $\mathrm{Rx}$ units (i.e., $\theta_{\mathrm{TX}}=0^{\circ}$ ) and $\mathrm{b}$ ) some shift in the orientation of Tx (i.e., $\theta_{\mathrm{TX}}=10^{\circ}$ ). In the NLOS scenario (see Fig. 4.b), transmitter and receiver do not look at each other and the transmission depends only on the reflected light from a whiteboard. $d_{w}$ denotes the distance between the whiteboard (used as a reflector) and Tx-Rx front-ends.

In Fig. 5, we present the received constellations and eye diagrams for LOS scenario. We assume strict alignment between transmitter and receivers and set $d=1 \mathrm{~m}$. From Fig. 5.a, it is observed that received constellation is noisy and the eye diagram is nearly closed when no lens is used. In order to improve the performance, we use a $24.5 \mathrm{~mm}$ plano-convex lens at Tx front-end to collimate the transmitted light pattern. This consequently increases the received SNR and gives better results as can be observed in eye diagrams presented in Fig. 5.b. 


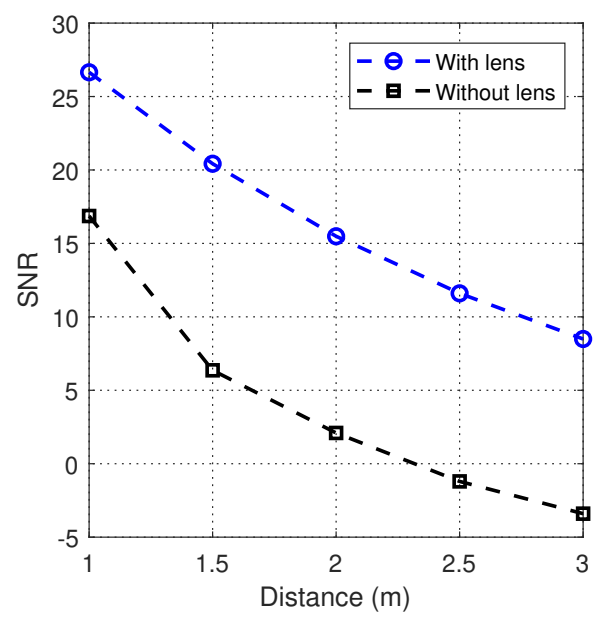

(a)

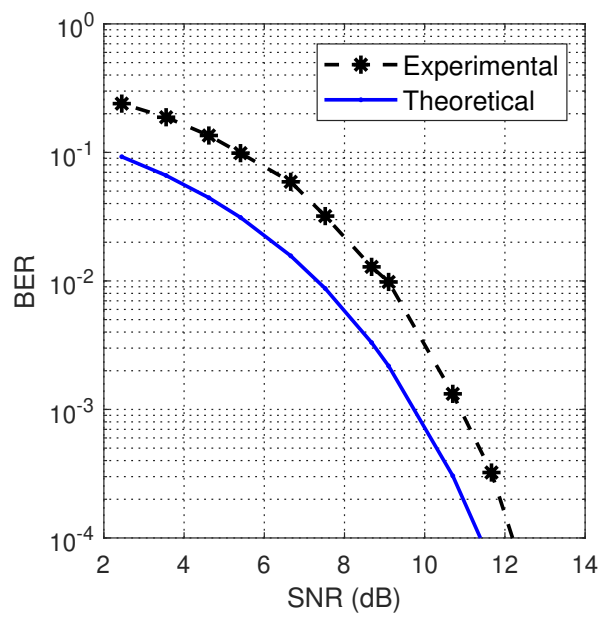

(b)

Fig. 6. Experimental measurements: (a) estimated SNR versus distance (b) measured BER versus SNR.

In Fig. 6, we present SNR and BER performance versus distance. We assume LOS scenario with strict alignment where $d$ varies between $1 \mathrm{~m}$ and $3 \mathrm{~m}$ with a step size of $0.5 \mathrm{~m}$. The estimate of SNR is obtained based on (16) in conjunction with (14) and (15) where noise power and effective channel are estimated. In Fig. 6.a, it can be observed that at a distance of $1 \mathrm{~m}, 16.87 \mathrm{~dB}$ SNR is obtained if no lens is used. This decreases to $-3.4 \mathrm{~dB}$ at $d=3 \mathrm{~m}$. When a plano-convex lens is used at the transmitter, some improvements are observed. Specifically, we obtain SNR values of $26.65 \mathrm{~dB}$ and $8.49 \mathrm{~dB}$ at $d=1 \mathrm{~m}$ and $d=3 \mathrm{~m}$ respectively. These indicate SNR improvements of around $9.8 \mathrm{~dB}$ and $11.89 \mathrm{~dB}$ in comparison to no lens case.

For BER measurements, we transmit $N_{F}=200$ frames for each SNR value. Each frame includes $L_{D}=8000$ bit per frame (see Table I). Therefore, the total number of transmitted bits is $N_{F} L_{D}=200 \times 8000=1.6 \times 10^{6}$ bits. We compare the transmitted and received bits and present the BER for each SNR value in Fig. 6.b. As a benchmark,

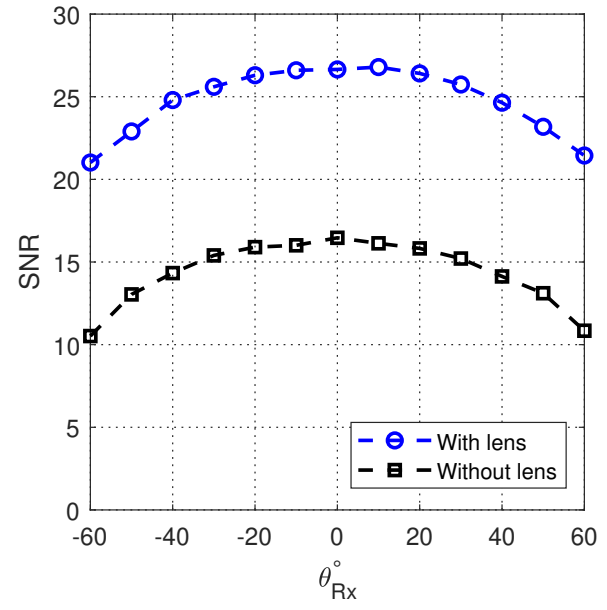

(a)

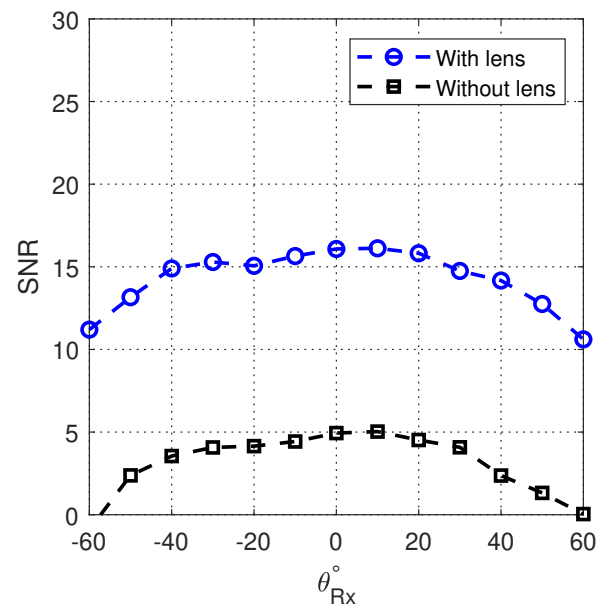

(b)

Fig. 7. Receiver SNR versus reception angle for LOS scenario: (a) $\theta_{\mathrm{TX}}=0^{\circ}$ (b) $\theta_{\mathrm{TX}}=10^{\circ}$

we further include the analytical BER expression for OOK given by $B E R=Q(\sqrt{\mathrm{SNR}})$ [16]. It can be readily observed that theoretical and experimental curves have a close match. There is about $1.8 \mathrm{~dB}$ shift between the two curves as a result of inaccuracy associated with symbol timing, frame synchronization and channel estimation. As SNR increases, the shift between these two curves decreases since estimation accuracy relatively improves.

In Fig. 7, we investigate the effect of transmitter orientation for the LOS scenario. We consider $d=1 \mathrm{~m}$ and present SNR versus reception angle. For transmitter orientation, we assume $\theta_{\mathrm{TX}}=0^{\circ}$ (Fig. 7.a) and $\theta_{\mathrm{TX}}=10^{\circ}$ (Fig.7.b). As expected, it can be observed from Fig. 7.a that the maximum SNR is observed at $\theta_{\mathrm{RX}}=0$ and SNR decreases as $\left|\theta_{\mathrm{RX}}\right|$ increases. In Fig. 7.b. where we set $\theta_{\mathrm{TX}}=10^{\circ}$, the maximum SNR is obtained at $\theta_{\mathrm{RX}}=10$. In both cases, the employment of lens increases the received SNR by around $10 \mathrm{~dB}$.

In Fig. 8, we investigate the effect of transmitter orientation for the NLOS scenario. The transmitter and receiver are 


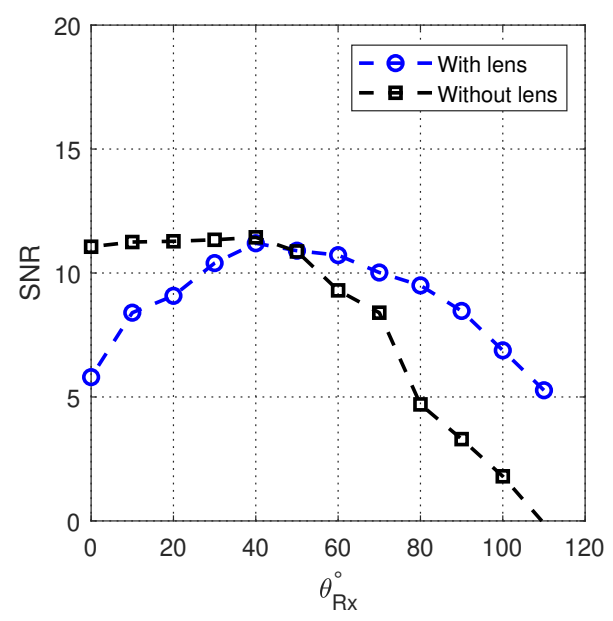

(a)

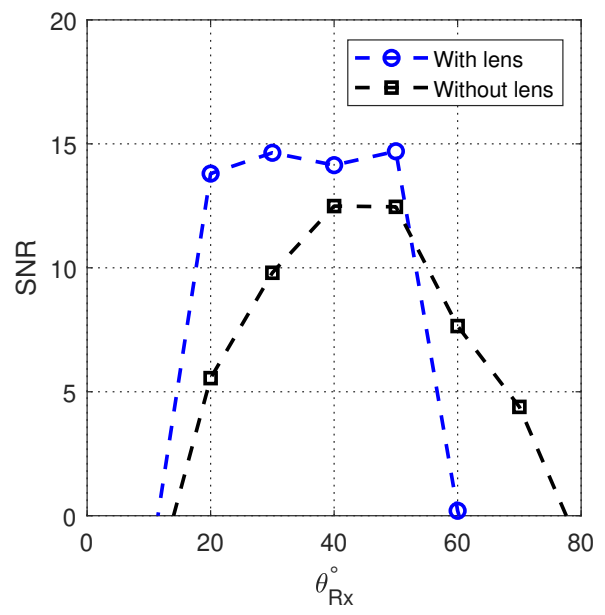

(b)

Fig. 8. Receiver SNR versus reception angle for NLOS scenario at $d_{w}=$ $0.5 \mathrm{~m}$ : (a) $\theta_{\mathrm{TX}}=60^{\circ}$ (b) $\theta_{\mathrm{TX}}=90^{\circ}$.

located at an equal distance from the reflecting surface with $d_{w}=0.5 \mathrm{~m}$. We present SNR versus reception angle. It is observed that for $\theta_{\mathrm{TX}}=60^{\circ}$ (Fig. 8.a), the received power is relatively low when the receiver orientation angle is less than $\theta_{\mathrm{RX}}=40^{\circ}$ assuming the employment of lens. Without a lens, PD provides a wider FOV which gains more power from the reflected rays even for values of $\theta_{\mathrm{RX}}$ smaller than. At $\theta_{\mathrm{TX}}=90^{\circ}$, the receiver with lens starts to get signal at almost $\theta_{\mathrm{RX}}=20^{\circ}$. It reaches the maximum at $50^{\circ}$ degrees, then it starts to degrade dramatically again. Without any lens, when the receive orientation angle is larger than $\theta_{\mathrm{RX}}=50^{\circ}$, $\mathrm{PD}$ receives more power from the reflected rays.

\section{CONCLUSION}

In this paper, we have experimentally evaluated the performance of a software-defined VLC system. The experimental set-up was built upon on a pair of modified USRPs where RF daughterboards are replaced by baseband LFTX and LFTX cards and a custom design front-end. In LOS scenario, we varied the transmission distance between 1 to 3 meters with a step size of $0.5 \mathrm{~m}$. For each point, we measured SNR and corresponding BER with and without a lens. For a transmit power of $0 \mathrm{~dB}$, we obtained SNR $=16.87 \mathrm{~dB}$ at a distance of $1 \mathrm{~m}$ without any lens. With the plano-convex lens at the transmitter, SNR increased to $26.65 \mathrm{~dB}$. We further investigated NLOS scenarios where there is no LOS component and transmission relies only on reflected components. $\mathrm{Up}$ to $\mathrm{SNR}=14.7 \mathrm{~dB}$ was obtained for NLOS scenarios. It was further observed that even for low values of transmitter/receiver orientation angles, a sufficiently high SNR (around $14 \mathrm{~dB}$ at $\theta_{\mathrm{RX}}=20^{\circ}$ ) can be obtained.

\section{REFERENCES}

[1] S. Dimitrov and H. Haas, Principles of LED Light Communications: Towards Networked Li-Fi. Cambridge, U.K.: Cambridge Univ. Press, 2015, ch. 2.

[2] M. Uysal, C. Capsoni, Z. Ghassemlooy, A. Boucouvalas, and E. Udvary, Optical Wireless Communications: An Emerging Technology. Springer, 2016.

[3] P. H. Pathak, X. Feng, P. Hu, and P. Mohapatra, "Visible light communication, networking, and sensing: A survey, potential and challenges," IEEE Communications Surveys Tutorials, vol. 17, no. 4, pp. 2047-2077, Fourthquarter 2015.

[4] T. T. Son, A. Burton, H. Le-Minh, and D. Q. Hien, "Experimental study of pc-to-pc over a visible light channel using li-fi usb dongle," in 2019 26th International Conference on Telecommunications (ICT), April 2019, pp. 215-219.

[5] D. Tsonev, S. Videv, and H. Haas, "Unlocking spectral efficiency in intensity modulation and direct detection systems," IEEE J. Sel. Areas Commun, vol. 33, no. 9, pp. 1758-1770, 2015.

[6] H. Durrani, J. Kasi, M. Sohail, M. Bokhari, and A. Kasi, "Implementation of unipolar OFDM based VLC transmission system under dimming constraint for high speed data through FPGA," J. Comput. Commun. Instrum. Engg., vol. 5, no. 1, pp. 14-17, 2018.

[7] S. M. Mana, P. Hellwig, J. Hilt, P. W. Berenguer, and V. Jungnickel, "Experiments in non-line-of-sight Li-Fi channels," in 2019 Global LIFI Congress (GLC), June 2019, pp. 1-6.

[8] Z. Dong, K. Cui, G. Chen, and Z. Xu, "Non-line-of-sight link performance study for indoor visible light communication systems," in Proc. of SPIE, Free Space Laser Communications IX, August 2010.

[9] Z. Lu, "Experimental demonstration of non-line-of-sight visible light communication with different reflecting materials using a gan-based micro-led and modified ieee 802.11ac," in AIP Advances, vol. 8, no. 105017, 2018, pp. 1-9.

[10] W. Hussain, H. F. Ugurdag, and M. Uysal, "Software defined VLC system: Implementation and performance evaluation," in 2015 4th International Workshop on Optical Wireless Communications (IWOW), Sep. 2015, pp. 117-121.

[11] O. R. B. Sayco and A. C. Gordillo, "Design and implementation for a USRP - based visible light communications transceiver," in 2019 UNSA International Symposium on Communications (UNSA ISCOMM), March 2019, pp. 1-5.

[12] Ettus Research, http://www.ettus.com, [Accessed: January 20, 2020].

[13] LiFi R\&D kit, http://www.hyperiontechs.com/wpcontent/uploads/2019/07/datasheet.pdf, [Accessed: January 20, 2020].

[14] R. W. H. Jr., Introduction to Wireless Digital Communication: A Signal Processing Perspective. Englewood Cliffs, NJ, USA: Prentice-Hall,, Mar. 2017.

[15] B. Aly, M. Elamassie, M. Uysal, and E. Kınav, "Experimental evaluation of unipolar OFDM VLC system on software defined platform," in 2019 15th International Conference on Telecommunications (ConTEL), July 2019, pp. 1-6.

[16] Z. Ghassemlooy, W. Popoola, and S. Rajbhandari, Optical Wireless Communications: System and Channel Modelling with MATLAB. CRC Press, 2017. 